\title{
COLLECTIONWISE NORMALITY IN SCREENABLE SPACES
}

\author{
MARY ELLEN RUDIN
}

\begin{abstract}
We show that if there is any normal screenable space which is not paracompact, there is one which is not collectionwise normal.
\end{abstract}

We use space to mean a Hausdorff topological space.

If a normal Moore space fails to be paracompact (and metric) it does so because it it not collectionwise normal [1]. If a normal screenable space fails to be paracompact, it does so because it is not countably paracompact (or countably metacompact) [2]. A recent (consistency) example of a normal screenable nonparacompact space is collectionwise normal [3], and a natural observation of Tall is that normal screenable spaces are collectionwise normal with respect to paracompact sets. Thus the following question arose: are all normal screenable spaces collectionwise normal? The purpose of this note is to prove that the answer is no, assuming the existence of any normal screenable nonparacompact space $X$. Imitating the construction of “Bing's $G$ " [4] we construct a normal screenable space consisting of some isolated points together with a closed discrete family of $\omega_{1}$ copies of $X$ which cannot be contained in disjoint open sets. We thus construct a machine which, for each $X$, constructs a $G^{*}$ as follows:

I. Assume that $X$ is a normal screenable nonparacompact space.

Let $S=\{s: X \rightarrow[0,1] \mid s$ is continuous $\}$.

Let $Q=\left\{A: \omega_{1} \rightarrow S\right\}$.

Let $G=\{g: Q \rightarrow[0,1]\}$.

For all $\alpha \in \omega_{1}$ and $x \in X$, let $f_{\alpha x}$ be the term of $G$ defined by $f_{\alpha x}(A)=A(\alpha)(x)$; let $F_{\alpha}=\left\{f_{\alpha x} \mid \alpha \in \omega_{1}, x \in X\right\}$ and $F=\cup\left\{F_{\alpha} \mid \alpha \in \omega_{1}\right\}$.

A subbasic open set in the product topology on $G$ is of the form $\{g \in G \mid g(A) \in$ $B$ ) for some $A \in \mathbb{Q}$ and open $B$ in $[0,1]$.

We topologize $G$ by using basic open sets from the product topology as basic open sets in $G$ for points of $F$ and declaring each point of $(G-F)$ to be isolated. This topology is certainly Hausdorff since it refines the product topology.

II. Since singletons in $G-F$ are open, $F$ is closed. If $f_{\alpha x} \in U=\{g \in G \mid g(A) \in$ $B$ for some $A \in \mathbb{Q}$ and open $B$ in $[0,1]$, then $A(\alpha)(x) \in B$ and, if $V=A(\alpha)^{-1}(B)$, then $V$ is open in $X$ and $\left\{f_{\alpha y} \mid y \in V\right\} \subset U$. Similarly, if $x \in V$, open in $X$, there is an $s \in S$ with $x \in s^{-1}([0,1)) \subset V$. Define $A \in \mathbb{Q}$ by $A(\alpha)=s$ and $A(\beta)(x)=1$ for

Received by the editors April 13, 1982 and, in revised form, June 18, 1982.

1980 Mathematics Subject Classification. Primary 54D15, 54G20, 54E65: Secondary 03E35.

Key words and phrases. Collectionwise normal, normal, screenable, paracompact.

i 1983 American Mathematical Society $0002-9939 / 82 / 0000-0692 / \$ 01.75$ 
all $\beta \neq \alpha$ and $x \in X$. Then $(\{g \in G \mid g(A) \in[0,1)\} \cap F) \subset\left\{f_{\alpha y} \mid y \in V\right\} \subset F_{\alpha}$. Thus the map from $F_{\alpha}$ to $X$ defined by $f_{\alpha x} \rightarrow x$ is a homeomorphism. Also $F_{\alpha}$ is both open and closed in $F$ and $\left\{F_{\alpha} \mid \alpha \in \omega_{1}\right\}$ is thus a closed discrete family in $G$.

LEMMA 1. G is normal.

Proof. Suppose $H$ and $K$ are disjoint closed sets in $G$. We want disjoint open sets containing $H$ and $K$, respectively. Since the points of $G-F$ are isolated we can assume that $(H \cup K) \subset F$. Since $X$ is normal and the map $f_{\alpha x} \rightarrow x$ is a homeomorphism, for each $\alpha \in \omega_{1}$, we can choose a continuous $s_{\alpha}: X \rightarrow[0,1]$ with $s_{\alpha}\left(\left\{x \in X \mid f_{\alpha x} \in H\right\}\right)=0$ and $s_{\alpha}\left(\left\{x \in X \mid f_{\alpha x} \in K\right\}\right)=1$. Define $A \in Q$. by $A(\alpha)=$ $s_{\alpha}$. Then $H \subset U=\left\{g \in G \mid g(A) \in\left[0, \frac{1}{2}\right)\right\}$ and $K \subset V=\left\{g \in G \mid g(A) \in\left(\frac{1}{2}, 1\right]\right\}$. Since $U \cap V=\varnothing$ and $U$ and $V$ are open in $G$, the proof of Lemma 1 is complete.

III. Our desired screenable normal not collectionwise normal space $G^{*}$ will be a closed subset of $G$.

Since $X$ is normal, but not countably paracompact [5], there is an open cover $\left\{O_{n} \mid n \in \omega\right\}$ of $X$ such that, if $D_{n}=O_{n}-\cup_{m<n} O_{m}$ and $T_{n}$ is open and $T_{n} \supset D_{n}$, then $\left\{T_{n} \mid n \in \omega\right\}$ is not point finite.

If $n \in \omega$ and $\alpha \in \omega_{1}$, let $\mathbb{Q}_{\alpha n}$ be the set of all $A$ in $Q$ such that

(1) $A(\beta)(x)=1$ for all $\beta \neq \alpha$ in $\omega_{1}$ and $x \in X$, and

(2) $A(\alpha)^{-1}([0,1))$ intersects $D_{n}$ and is contained in $O_{n}$.

Let $Q_{\alpha}=\cup\left\{\Theta_{\alpha n} \mid n \in \omega\right\}$.

We let $G^{*}$ be the set of all $g$ in $G$ such that

(1) if $n \in \omega$, there is at most one $\alpha \in \omega_{1}$ with $g(A) \in[0,1)$ for some $A \in \hat{\mathbb{t}}_{\alpha n}$, and

(2) if $g(A)$ and $g\left(A^{\prime}\right)$ are in $[0,1)$ for some $A$ and $A^{\prime}$ in $\mathcal{Q}_{\alpha}$, then $A(\alpha)^{-1}([0,1)) \cap$ $A^{\prime}(\alpha)^{-1}([0,1)) \neq \varnothing$.

Observe that $F \subset G^{*}$. Since the points of $G-F$ are isolated, $G^{*}$ is a closed (and hence normal) subset of $G$.

LEMMA 2. $G^{*}$ is screenable.

Proof. Suppose that $U$ is an open cover of $G^{*}$. We want to construct a $\sigma$-disjoint refinement $V$ of $U$ covering $F$. Then $V \cup\left\{\{g\} \mid g \in G^{*}-F\right\}$ will be a $\sigma$-disjoint refinement of $U$ covering $G^{*}$.

Fix $\alpha \in \omega_{1}$. For each $x \in X, f_{\alpha x} \in U_{x} \in Q_{1}$. Since $x \in D_{n}$ for some $n$, there is an $A_{x} \in \mathbb{Q}_{\alpha n}$ with $x \in T_{x}=\left\{y \in X \mid A_{x}(\alpha)(y) \in[0,1)\right\} \subset\left\{y \in X \mid f_{\alpha y} \in U_{x}\right\}$. Since $X$ is screenable there is a $\sigma$-disjoint refinement of of $\left\{T_{x} \mid x \in X\right\}$ covering $X$; say

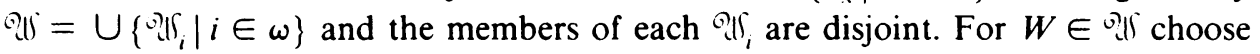
$x_{W} \in X$ with $W \subset T_{x_{u}}$ and let $U_{W}=\left\{g \in U_{x} \mid g\left(A_{x_{u}}\right) \in[0,1)\right\}$. For each $w \in W$ $\in$ Qlli, choose $A_{w W} \in \mathbb{Q}_{\alpha}$ such that $w \in A_{w \cdot W}(\alpha)^{-1}[0,1) \subset W$. Let $V_{w W}=\left\{g \in G^{*} \mid\right.$ $\left.g\left(A_{w^{\prime} W}\right) \in[0,1)\right\}$. Then, for $W \in$ ol $)$, define $W^{*}=U_{w^{\prime}} \cap\left(\cup\left\{V_{w \cdot W} \mid w \in W\right\}\right)$. Define "ill ${ }_{\alpha i n}=\left\{W \in \mathcal{O}_{i} \mid x_{W} \in D_{n}\right\}$ and $\mathcal{V}_{\alpha i n}=\left\{W^{*} \mid W \in \mathcal{O}_{\alpha i n}\right\}$.

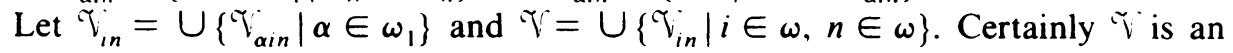
open cover of $F$ refining $\mathcal{Q}$. We claim that the members of each $\tau_{i n}$ are disjoint and thus $\widetilde{T}$ is $\sigma$-disjoint. 
To see this, first suppose that for some $\alpha, W$ and $W^{\prime}$ are in $\mathscr{W}_{\alpha i n}$. If $w \in W$ and $w^{\prime} \in W^{\prime}, A_{w W}$ and $A_{w^{\prime} W^{\prime}}$ belong to $\mathcal{Q}_{\alpha} ;$ and $A_{w W}(\alpha)^{-1}[0,1) \subset W, A_{w^{\prime} W^{\prime}}(\alpha)^{-1}[0,1)$ $\subset W^{\prime}$, and $W \cap W^{\prime}=\varnothing$. So, by (2) in the definition of $G^{*}$, no $g \in G^{*}$ is in $\left(V_{w W} \cap V_{w^{\prime} W^{\prime}}\right)$; hence $W^{*} \cap W^{*}=\varnothing$.

Next, suppose $W \in \mathcal{Q} \int_{\alpha i n}$ and $W^{\prime} \in \mathcal{U} \int_{\beta i n}$ for some $\alpha \neq \beta$ in $\omega_{1}$. Since $A_{x_{w}} \in \mathcal{U}_{\alpha n}$ and $A_{X_{u}} \in \mathcal{Q}_{\beta n}$ (the $A_{x_{W}}$ being defined with $\beta$ rather than $\alpha$, fixed), by (1) of the definition of $G^{*}$, there is no $g \in G^{*}$ in $U_{W} \cap U_{W^{\prime}}$. Thus $W^{*} \cap W^{*}=\varnothing$; and our proof that the members of $\tau_{i n}$ are disjoint is complete.

\section{LEMMA 3. $G^{*}$ is not collectionwise normal.}

Proof. Suppose that $F_{\alpha} \subset U_{\alpha}$, open in $G^{*}$. We show that $\left\{U_{\alpha} \mid \alpha \in \omega_{1}\right\}$ are not disjoint.

Choose a countable basis 6 for $[0,1]$.

Fix $\alpha \in \omega_{1}$. If $x \in X$, there is a finite set $P_{x}$ of pairs $\langle A, B\rangle$ with $A \in \mathcal{A}^{\prime}$ and $B \in \beta$ such that $f_{\alpha x} \in U_{x}=\left\{g \in G^{*} \mid g(A) \in B\right.$ for all $\left.\langle A, B\rangle \in P_{x}\right\} \subset U_{\alpha}$. Without loss of generality we assume for all $\langle A, B\rangle$ in $P_{x}, 1 \notin B$ unless $A(\alpha)(x)=1$. Let $T_{x}=\left\{y \in X \mid A(\alpha)(y) \in B\right.$ for all $\left.\langle A, B\rangle \in P_{x}\right\}$ and $M_{x}=\{m \in \omega \mid$ there is $\langle A, B\rangle \in P_{x}$ with $A \in \mathbb{Q}_{\alpha m}$ and $\left.B \subset[0,1)\right\}$. By our definition of $D_{n}$, there are $x$ and $y$ in $X$ with $x \in T_{v}$ and $y \in D_{n}$ for some $n>\left(\sup M_{x}\right)$. Observe that since $y \in D_{n}$, if $m \in M_{v}$, then $m \geqslant n$; so $M_{v} \cap M_{x}=\varnothing$. Let $x_{\alpha}$ and $y_{\alpha}$ denote this chosen $x$ and $y$ for our fixed $\alpha$ and $P_{\alpha x}, P_{\alpha y}, M_{\alpha x}$, and $M_{\alpha \nu}$ the associated $P_{x}, P_{y}, M_{x}$ and $M_{y}$.

By the standard $\Delta$-system argument, one can find an uncountable subset $\Delta$ of $\omega_{1}$ such that

(1) for all $\alpha \in \Delta$, all $M_{x_{\alpha}}$ are the same set which we call $M_{x}$ and all $M_{y_{\alpha}}$ 's are the same set which we call $M_{y}$;

(2) there are $i$ and $j$ in $\omega$ such that, for all $\alpha \in \Delta$, the $P_{\alpha x}$ 's all have $i$ terms $\left\langle A_{\alpha 0}, B_{\alpha 0}\right\rangle, \ldots,\left\langle A_{\alpha(i-1)}, B_{\alpha(i-1)}\right)$, and the $P_{\alpha,}$ 's all have $j$ terms $\left\langle A_{\alpha 0}^{\prime}, B_{\alpha 0}^{\prime}\right\rangle, \ldots,\left\langle A_{\alpha(j-1)}^{\prime}, B_{\alpha(j-1)}^{\prime}\right\rangle$;

(3) for $\alpha \in \Delta$, if $k<i$, all $B_{k \alpha}$ 's are the same set, called $B_{k}$, and, if $l<j$, all $B_{l,}^{\prime}$,'s are the same set, called $B_{l}^{\prime}$;

(4) there are subsets $I$ of $i$ and $J$ of $j$ such that, for all $\alpha \in \Delta$, if $k \in I$, all $A_{\alpha k}$ 's are the same and, if $l \in J$, all $A_{\alpha l}^{\prime}$ 's are the same. And if $A=A_{\alpha k}$ for some $k \in i-I$ or $A=A_{\alpha l}^{\prime}$ for some $l \in j-J$, then $A$ is neither $A_{\beta h}$ for some $\beta \neq \alpha$ and $h<i$ nor $A_{\beta m}$ for some $\beta \neq \alpha$ and $m<j$.

Choose $\alpha \neq \beta$ in $\Delta$. Then define $g \in G$ by

(1) $g(A)=1$ if $A \notin\left\{A_{\alpha k} \mid k<i\right\} \cup\left\{A_{\beta l}^{\prime} \mid l<j\right\}$;

(2) $g(A)=A(\alpha)\left(x_{\alpha}\right)$ if $A=A_{\alpha k}$ for some $k<i$;

(3) $g(A)=A(\beta)\left(y_{\beta}\right)$ if $A \in\left\{A_{\beta l}^{\prime} \mid l<j\right\}-\left\{A_{\alpha k} \mid k<i\right\}$.

If we can show that $g \in U_{\alpha} \cap U_{\beta}$ our proof is complete. By (2), if $g \in G^{*}, g \in U_{\alpha}$. If for some $l<j$ and $k<i, A_{\beta l}^{\prime}=A_{\alpha k}$, then $A_{\alpha k}=A_{\beta k}$ and $A_{\beta l}^{\prime}=A_{\alpha l}^{\prime}$ and, since $x_{\alpha} \in T_{y_{\alpha}}, A_{\alpha l}^{\prime}(\alpha)\left(x_{\alpha}\right) \in B_{l}^{\prime}$. Thus, since $g\left(A_{\beta l}^{\prime}\right)=A_{\alpha k}(\alpha)\left(x_{\alpha}\right)=A_{\alpha l}^{\prime}(\alpha)\left(x_{\alpha}\right), g\left(A_{\beta l}^{\prime}\right)$ $\in B_{l}^{\prime}$. This, together with (3), shows that, if $g \in G^{*}, g \in U_{\beta}$. So it remains to prove that, $g \in G^{*}$. 
If $g(A) \in[0,1)$ for any $A \in \mathbb{Q}_{\delta}$, then $\delta$ is $\alpha$ or $\beta$ and $A \in\left\{A_{\alpha h} \mid h<i\right\} \cup\left\{A_{\beta l}^{\prime} \mid l\right.$ $<j\}$.

If, for some $h<i$ and $m \in \omega, A_{\alpha h} \in \mathbb{Q}_{\alpha m}$ and $g\left(A_{\alpha h}\right) \in[0,1)$, then $m \in M_{x}$. If $l<j, m \in \omega, A_{\beta l}^{\prime} \in \mathbb{Q}_{\beta m}$, and $g\left(A_{\beta l}^{\prime}\right) \in[0,1)$, then, certainly, $m \in M_{y}$ unless $A_{\beta l}^{\prime}=$ $A_{\alpha k}$ for some $k<i$. In this case, as shown above, $g\left(A_{\beta l}^{\prime}\right) \in B_{l}^{\prime}$, and thus $m \in M_{y}$. Since $M_{x} \cap M_{y}=\varnothing, g$ satisfies (1) of the definition of $G^{*}$.

If $g\left(A_{\alpha h}\right) \in[0,1)$ for some $h<i, x_{\alpha} \in A_{\alpha h}(\alpha)^{-1}[0,1)$; so (2) in the definition of $G^{*}$ is satisfied for any $A$ and $A^{\prime}$ in $\mathbb{Q}_{\alpha}$.

If $g(A) \in[0,1)$ for some $A \in \mathbb{Q}_{\beta}$, then $A$ is $A_{k l}^{\prime}$ for some $l<j$. If $g(A)=A(\alpha)\left(y_{\beta}\right)$, then, since $x_{\beta} \in T_{y_{\beta}}, x_{\beta} \in A(\beta)^{-1}[0,1)$. Otherwise, $A=A_{\alpha k}$ for some $k<i$ and $g(A)=A(\alpha)\left(x_{\alpha}\right)$ and $A_{\alpha k}=A_{\beta k}$. Since $g(A) \neq 1,1 \notin B_{k}$, and $x_{\beta} \in A(\beta)^{-1}[0,1)$. So (2) in the definition of $G^{*}$ is satisfied for all $A$ and $A^{\prime}$ in $Q_{\beta}$ and the proof of our lemma is complete.

\section{BIBLIOGRAPHY}

I. R. H. Bing, Metrization of topological spaces, Canad. J. Math. 3 (1951), 175-186.

2. K. Nagami, Paracompactness and strong screenability, Nagoya Math. J. 88 (1955), 83-88.

3. M. E. Rudin, A normal screenable nonparacompact space. General Topology Appl. 14 (1982), 1-116.

4. C. H. Dowker, On countably paracompact spaces, Canad. J. Math. 3 (1951), 219-224.

Department of Mathematics, University of Wisconsin, Madison, Wisconsin 53706. 